\title{
What will follow the first hundred thousand genomes in the NHS?
}

Malcolm Grant ${ }^{*}, 1$ \& John Paul Maytum ${ }^{1}$

${ }^{1} \mathrm{NHS}$ England, Skipton House, 80 London Road, SE1 6LH London, UK

*Author for correspondence: Tel.: 0113825 1104; Malcolm.Grant@nhs.net

\section{"over time we expect that the Genomic Medicine Service will grow from its base in cancer and rare disease to driving prescribing and treatment optimization and personalization of interventions"}

First draft submitted: 8 March 2018; Accepted for publication: 8 March 2018; Published online: 20 June 2018

Keywords: genomics $\bullet$ personalized medicine $\bullet$ policy issues

The 100,000 Genomes Project has been the first genomics initiative in the world to recruit patients through routine care and offer treatment through routine channels. As recruitment to the project concludes at the end of this year, England's National Health Service is rolling out a comprehensive National Genomic Medicine Service offering broad and equitable access to testing and treatment for the country's entire 55 million population.

It is increasingly acknowledged that genomics has the potential to transform future medical practice, providing more precise, personalized diagnosis and treatment while also providing potential for significant new approaches to improve population health [1]. Consequently, across the globe, countries are undertaking a wide range of genomic and personalized medicine initiatives to realize this potential, and determine how to best apply them within day-to-day medical practice [2].

Unlike many other research initiatives, the UK's 100,000 Genomes Project was developed through a unique partnership with the National Health Service (NHS), with a clear and deliberate focus on recruiting patients from across the country with common cancers and rare diseases through routine care [3]. This focus was intentional because we always had our eyes on the bigger prize - supporting the widespread utilization of genomics across a population health system. As a result, the Project is enabling the NHS to embed genomics more systemically into routine care. For example in cancer, the hospitals involved are establishing genomic-friendly pathways and changing the way tissue and samples are collected and handled, moving away from using formalin to fresh-frozen pathways.

To deliver the Project, NHS England established a network of NHS Genomic Medicine Centers to identify and enroll suitable patients (enabling recruitment to be drawn from across the country's diverse population), to support and implement experimental approaches to provide high-quality DNA, and embed new genomic-friendly pathways and care approaches. Importantly, this was supported by a coordinated Genomics Education Program to support and upskill NHS staff to be able to fully utilize genomics in care.

The 100,000 Genomes Project has only been possible because of the nature of the NHS, a universal healthcare system caring for and following individuals from cradle to grave, with rich longitudinal health records, to enable the high-fidelity clinical phenotyping required to gather significant insight from genomic analysis.

Common standards, specifications and protocols have been created driving a commonality of approach and culture across the 100-plus hospital organizations involved that is rarely seen in healthcare provision - an essential component to enable the comparability of information needed to begin to release the true potential of genomics. Early results are demonstrating the potential benefits, with a four to fivefold increase in diagnostic yield for people with rare diseases and the identification of actionable variants in $65 \%$ of cancer patients.

Future Medicine 


\section{A national genomic medicine service}

Despite the successes, there is still more to be done to fully establish the application of genomic technologies across the NHS while ensuring equitable access. This is particularly true in primary care where new molecular point-of-care testing devices could provide real time data to guide more appropriate prescribing in a number of areas.

As the 100,000 Genomes Project is on track to complete by the end of 2018, the focus is now firmly on ensuring the UK continues to build on the progress and investment that has been made to date. To build on the legacy, in March 2017 NHS England's Board set out its plans to create a national Genomic Medicine Service that would begin operation in the autumn 2018 once recruitment to the Project was complete [4].

The Genomic Medicine Service will provide England's 55 million population with equitable access to consistently high-quality genetic and genomic testing, including whole genome sequencing, and will underpin the development of personalized medicine approaches across the system. The Genomic Medicine Service will evolve the role of existing clinical genetics services and the NHS Genomic Medicine Centres to enable seamless delivery for patients, and provide comprehensive access to specialist expertise from hospitals and specialist providers through primary care.

To ensure appropriate capacity and conformity to deliver the testing, NHS England is establishing a national network of seven genomic laboratory hubs working to agreed national standards. Each hub will be responsible for coordinating genomic testing for their geographical area, while a smaller number of hubs will take on responsibility for low-volume specialist testing.

A key part of the future approach will be a national genomic test directory, identifying the most appropriate test for each clinical indication and the technology by which it should be delivered. The directory will cover the full repertoire of genetic and genomic tests from single gene to whole genome sequencing, using the latest evidence about when whole genome sequencing would be clinically appropriate, affordable and cost-effective to provide a better outcome for patients.

The pace of change in this field is significant, so it is essential that the service is able to respond to future technological changes. Going forward it will be key that we collate and analyze information about the utilization of genomics and the outcomes generated to help inform the annual review of the directory and ensure the system is able to provide access for patients to the most clinically appropriate, affordable, cutting-edge technology.

\section{Genomic-led personalization}

The development of the NHS Genomic Medicine Service will be an ongoing evolution to 2025 and beyond; over time we expect that the Genomic Medicine Service will grow from its base in cancer and rare disease to driving prescribing and treatment optimization and personalization of interventions. Our aim is to realize the potential benefits of genomic-led personalization including earlier diagnosis, increasing treatment possibilities by identifying disease earlier, greater diagnostic yield, and more precise diagnosis, allowing the potential for better segmentation of conditions with increased treatment effectiveness through better treatment selection.

On the more immediate horizon, genomic characterization will enable more patients to be offered the opportunity to take part in clinical trials, through the improved characterization of condition and driver targets, alongside improved access to a range of prognostics and preventative approaches through the identification of predisposition markers.

Through an ongoing partnership between the NHS and Genomics England it will be possible to secure significant benefits for research, development and discovery in both academia and the life sciences industry which we intend to leverage on behalf of the NHS, taxpayers and the wider economy. We want to ensure we create an environment where NHS patients are first in line to benefit from the development of new treatments or interventions.

\section{The importance of patient \& participant involvement}

I have spoken a lot about the role and importance of the NHS, but it goes without saying that all of our achievements have only been possible because of the active involvement and consent of all of the people who have volunteered to take part, coupled with the support and understanding of our broader society. This has not arisen by accident but has been driven by ongoing engagement and knowledge-sharing by the Project partners, building on the public's trust in the NHS and understanding of the culture of clinical research that keeps the NHS at the forefront of technology. 
In such a fast-moving world, the support for genomics is not unquestioning and we recognize the ongoing need to invest time and effort to grow and nurture this support as the technology and its use advances [5,6]. It is paramount that as we develop our future approach we retain and ensure the public's trust and confidence in the consent and security of patient data.

We know that we have only started to scratch the surface of the transformative power of genomic medicine. There is still a huge amount of discovery to be undertaken. I believe that we will continue to see a reduction in the cost of sequencing the whole genome, which will further accelerate and enhance the pace of discovery and deliver greater patient benefits.

Within the next decade we will see a new taxonomy of medicine based on an understanding of underlying drivers of a patient's condition and their individual response. There will be routine use of whole genome sequencing and new genomic technologies across the breadth of clinical pathways, with genomics a foundation across many if not all clinical roles. This is why a national Genomic Medicine Service is the right next step for the NHS and why we are determined to maintain our world-leading position in the use of these technologies.

\section{Financial \& competing interests disclosure}

The authors have no relevant affiliations or financial involvement with any organization or entity with a financial interest in or financial conflict with the subject matter or materials discussed in the manuscript. This includes employment, consultancies, honoraria, stock ownership or options, expert testimony, grants or patents received or pending, or royalties.

No writing assistance was utilized in the production of this manuscript.

\section{Crown copyright}

This work is licensed under a Crown Copyright protection and licensed for use under the Open Government License unless otherwise indicated. Where any of the Crown copyright information in this work is republished or copied to others, the source of the material must be identified and the copyright status under the Open Government License acknowledged.

\section{References}

1. Brittain HK, Scott R, Thomas E. The rise of the genome and personalised medicine. Clin. Med. (Lond.) 17(6), 545-551 (2017).

2. Manolio TA, Abramowicz M, Al-Mulla F et al. Global implementation of genomic medicine: we are not alone. Sci. Transl. Med. 7(290), 290 ps13 (2015).

3. Caulfield M, Davies J, Dennys M et al. The 100,000 Genomes Project Protocol. Genomics England, UK (2017). https://doi.org/10.6084/m9.figshare.4530893.v2

4. Hill S. Introducing genomics into cancer care. Br. J. Surg. 105(2), e14-e15 (2018).

5. Parry V. Commit to talks on patient data and public health. Nature 548(7666), 137 (2017).

6. Department of Health. Chief medical officer annual report 2016: generation genome (2017). www.gov.uk/government/uploads/system/uploads/attachment_data/file/631043/CMO_annual_report_generation_genome.pdf 
